\title{
Erratum to: Analysis of the duodenal microbiotas of weaned piglet fed with epidermal growth factor-expressed Saccharomyces cerevisiae
}

Zhongwei Zhang ${ }^{1}$, Lili Cao ${ }^{2}$, Yan Zhou', Shujin Wang ${ }^{1,3,4^{*}}$ and Lin Zhou ${ }^{3^{*}}$

\section{Erratum}

Unfortunately, incorrect author affiliations were presented in the original version of this article [1]. These affiliations have now been corrected within this erratum.

An updated contact address for Shujin Wang has also been provided:

Human and Animal Physiology, Wageningen University, De Elst 1, 6708 WD Wageningen, The Netherlands.

\footnotetext{
Author details

'Department of Intensive Care Unit, West China Hospital, Sichuan University, Chengdu, Sichuan 610041, People's Republic of China. 'Medical School, Chengdu University, Chengdu, Sichuan 610041, People's Republic of China. ${ }^{3}$ Shenzhen Premix Inve Nutrition Co., LTD, Shenzhen 518103, People's Republic of China. ${ }^{4}$ Human and Animal Physiology, Wageningen University, De Elst 1, 6708 WD Wageningen, The Netherlands.
}

Received: 6 September 2016 Accepted: 9 September 2016 Published online: 31 October 2016

\section{Reference}

1. Zhang Z, et al. BMC Microbiol. 2016;16:166. doi:10.1186/s12866-016-0783-7.

\footnotetext{
*Correspondence: wangrui19880622@sina.com; zhouwei382@163.com

'Department of Intensive Care Unit, West China Hospital, Sichuan University,

Chengdu, Sichuan 610041, People's Republic of China

${ }^{3}$ Shenzhen Premix Inve Nutrition Co., LTD, Shenzhen 518103, People's

Republic of China

Full list of author information is available at the end of the article
} 